\title{
Erratum to: Statistical Analysis of the Results of 20 years of Activity of the International VLBI Service for Geodesy and Astrometry
}

\author{
Z. M. Malkin ${ }^{a, *}$ \\ ${ }^{a}$ Central (Pulkovo) Astronomical Observatory, Russian Academy of Sciences, St. Petersburg, 196140 Russia \\ *e-mail:malkin@gaoran.ru
}

Received November 23, 2021; revised November 23, 2021; accepted November 23, 2021

DOI: $10.1134 / \mathrm{S} 1063772922020032$

Paper [1] needs two refinements.

1. In the first row of the header of Table 2, the correct number of sources in the ICRF3 catalogues is the following: 4536 (ICRF3-S/X), 824 (ICRF3-K), and 678 (ICRF3-X/Ka) [2].

2. In the introduction to paper [1], it is written "This can be considered the beginning of the radio astrometry era, although the term itself appeared apparently in the early 1970s (the earliest paper containing this term found by the author was dated 1973)." After publication of the paper, the author found the text of the lecture given by Thomas Clark (NASA Goddard Space Flight Center) at the Institute of Applied Astronomy in 2005 [3]. In this lecture, Clark quoted his note written to Robert Coates on February 27,1969 , where he wrote: “... we hope to measure positions [of radio sources] to similar accuracies- $\sim 0.001$ seconds of arc. These measurements might best be called "Radio Astrometry".

Thus, the term radio astrometry appeared several years earlier than it was assumed in [1].

The original article can be found online at

https://doi.org/10.1134/S1063772920020043

\section{REFERENCES}

1. Z. M. Malkin, Astron. Rep. 64(2), 168 (2020).

2. P. Charlot, C. S. Jacobs, D. Gordon, S. Lambert, et al., Astron. and Astrophys. 644, id. A159 (2020).

3. T. A. Clark, Building the Geodetic VLBI Network. Some Personal Recollections of Tom Clark. Lecture given at Institute of Applied Astronomy (St. Petersburg, 2005), https://ivscc.gsfc.nasa.gov/meetings/tow2005/ Clark.Lec.pdf. 